\title{
Application of Air Coupled Acoustic Thermography (ACAT) for Inspection of Honeycomb Sandwich Structures
}

\author{
Joseph N. Zalameda ${ }^{a}$, William P. Winfree ${ }^{b}$, Charles G. Pergantis ${ }^{c}$, Daniel DeSchepper ${ }^{c}$, \\ and David Flanagan ${ }^{\mathrm{c}}$ \\ ${ }^{a}$ U. S. Army Research Laboratory, Vehicle Technology Directorate \\ Nondestructive Evaluation Sciences Branch MS 231 \\ NASA Langley Research Center 23681 \\ ${ }^{\mathrm{b}}$ Nondestructive Evaluation Sciences Branch MS 231 \\ NASA Langley Research Center 23681 \\ ${ }^{c}$ U.S. Army Research Laboratory \\ Weapons and Materials Research Directorate \\ Aberdeen Proving Ground, Maryland 21005
}

\begin{abstract}
The application of a noncontact air coupled acoustic heating technique is investigated for the inspection of advanced honeycomb composite structures. A weakness in the out of plane stiffness of the structure, caused by a delamination or core damage, allows for the coupling of acoustic energy and thus this area will have a higher temperature than the surrounding area. Air coupled acoustic thermography (ACAT) measurements were made on composite sandwich structures with damage and were compared to conventional flash thermography. A vibrating plate model is presented to predict the optimal acoustic source frequency. Improvements to the measurement technique are also discussed.
\end{abstract}

Keywords: thermal nondestructive evaluation, composite honeycomb inspection, core damage detection, composite delamination.

\section{INTRODUCTION}

Civil aviation aircraft, defense air vehicles, and space exploration structural designs are using more advanced composite sandwich structures. Sandwich structures are typically made by bonding thin composite skins (facings) with a lightweight core in between. These structures are lower in cost, lightweight, reparable and can be molded into complex shapes. Sandwich structures require advanced inspection systems. Of concern is the detection of skin damage, the bond between the skin and core, and damage to the core material. Ultrasound is a common inspection method for these structures, however this technology requires the use of a gel or water couplant. This inspection technology also requires contact with the structure and therefore curved surfaces are more difficult to inspect. In addition, coin tap testing or instrumented tapping is commonly used for inspection of sandwich structures because of its simplicity and ability to detect core damage. Manual coin tap testing technique is highly subjective and does not completely map out the damaged areas. Instrumented tapping can map out core damage areas, however this can be relatively time consuming as the single detector is scanned over a grid marked area. Thermography is an inspection technique that has shown good potential for inspection of honeycomb structures because of its rapid inspection capability using full field imagery [1]. 
Thermography inspection systems, using flash lamp heating, have the advantage of being noncontact and are able to detect a wide variety of defects that can affect the diffusion of heat into the structure. Core damage in sandwich structures is difficult to detect with flash lamp heating methods because heat flow into the core can be limited.

A new flaw detection technique based on an infrared imager and air coupled acoustic excitation using a wide area sound source has been developed. Unlike vibrothermography or sonic thermography, the energy source does not have to be mechanically coupled to the structure. Mechanical coupling of the large amplitude vibrations can be a significant issue because the transducer needs to be mounted securely and ideally the part should be isolated from any holders or fixtures [2]. All these parameters can affect the repeatability of the inspection. Another disadvantage is the potential for damaging the structure at the coupling point [3].

It is demonstrated that areas of damage on honeycomb sandwich structures, not normally detected with conventional flash thermography, are detectable with the air coupled acoustic thermography (ACAT) technique. This technique uses large area noncontact coupling of the acoustic sound energy, and hence is capable of wide-area examination. The induced temperature changes are small due to the impedance mismatch between air and composites or metals. The small temperature increases are detected using synchronous detection. A reduction in the out of plane stiffness increases the coupling efficiency of the acoustic energy. Weak areas act like heat sources and thereby allow detection of defective areas. An analytical model of a clamped circular plate is given, experimentally tested, and verified using the ACAT system.

\section{ACAT SYSTEM DESCRIPTION}

The ACAT system is shown in Figure 1. The system consists of an infrared camera operating in the 3-5 micrometer wavelength band, a computer with image data capture system, an acoustic source, and chamber. The chamber was used to contain some of the sound and to provide a stable thermal environment. The acoustic source consists of a function generator, dual channel power amplifier, and an array of four compression horn driver loudspeakers. The loudspeaker array was positioned approximately $11.5 \mathrm{~cm}$ from the sample. The thermal detection system consists of a computer connected to the infrared camera through a digital image acquisition card. During measurement the computer triggers the function generator, which is synchronized to the image acquisition card. The acquired data are composed of a series of 12 bit resolution digital images ( $256 \times 320)$ captured at $1 / 15$ of a second. For a typical measurement, 2400 images are acquired and the acoustic source is pulsed at $0.5 \mathrm{~Hz}$ for a total of 80 cycles. The acquired data is processed pixel by pixel in the time domain using a fast Fourier transform algorithm to produce magnitude images as a function of frequency. The sound pressure level in the laboratory was measured around $110 \mathrm{dBA} 5$ feet away from the array. The acoustic frequency was varied from $800-2,000 \mathrm{~Hz}$. A maximum in the sound pressure level within this range was used to determine an optimal heating frequency.

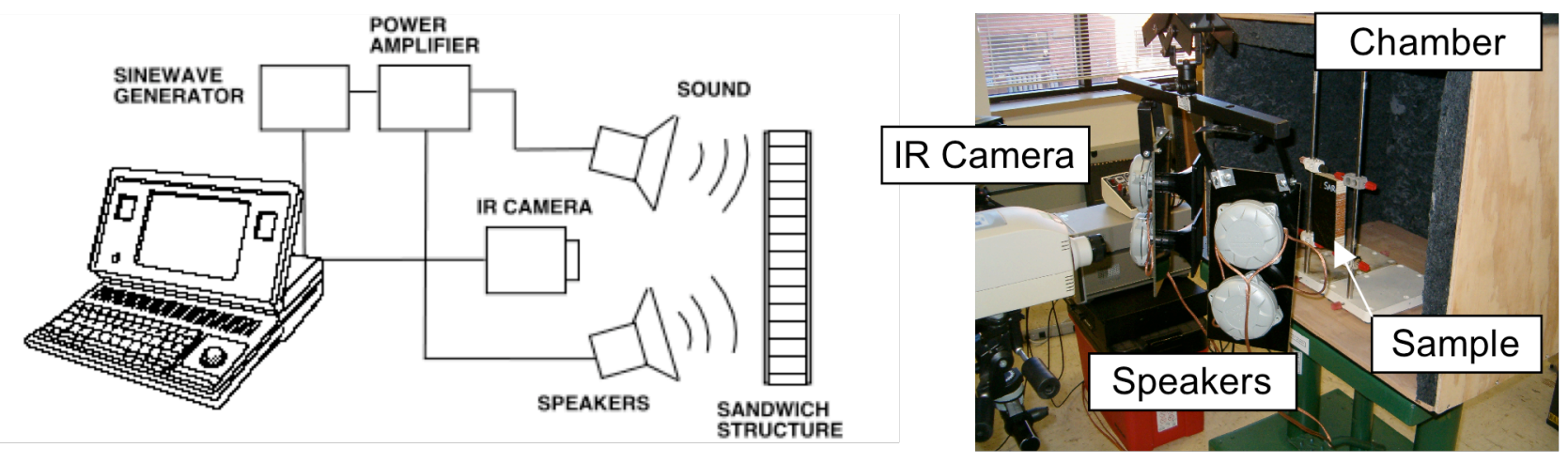

Figure 1. Air coupled acoustic thermography (ACAT) inspection system. 


\section{MODELING OF ACOUSTIC HEATING}

To model the acoustic heating, a sample with a suspended circular aluminum plate was measured. The sample consisted of a $0.32 \mathrm{~cm}$ thick 2024 aluminum plate with a $6.4 \mathrm{~cm}$ diameter hole. A $0.05 \mathrm{~cm}$ thick 2024 aluminum plate was bonded to the thicker plate using an epoxy adhesive thus allowing the thin layer to be suspended with the outer edges bonded to the thicker plate. A drawing and picture of the sample are shown in Figure 2. The normal modes of vibration for a circular plate are well known in the literature [4]. The modes of vibration are obtained from the fourth order wave equation:

$$
\nabla^{4} \mathrm{z}+\frac{3 \rho\left(1-\sigma^{2}\right)}{\mathrm{E} \mathrm{h}^{2}} \frac{\partial^{2} \mathrm{z}}{\partial \mathrm{t}^{2}}=0
$$

where $\mathrm{h}$ is the half thickness, $\mathrm{E}$ is the modulus of elasticity, $\sigma$ is the Poisson's ratio, $\rho$ is density and $\mathrm{z}$ is the out of plane displacement. The solution to the fourth order wave equation describes the modes of vibration. The modes of vibration or characteristic functions are obtained from [4] as:

Characteristic Functions $=\operatorname{Cos}(\mathrm{m} \theta)\left[\mathrm{J}_{\mathrm{m}}\left(\frac{\pi \beta_{\mathrm{mn}} \mathrm{r}}{\mathrm{a}}\right)-\frac{\mathrm{J}_{\mathrm{m}}\left(\pi \beta_{\mathrm{mn}}\right)}{\mathrm{I}_{\mathrm{m}}\left(\pi \beta_{\mathrm{mn}}\right)} \mathrm{I}_{\mathrm{m}}\left(\frac{\pi \beta_{\mathrm{mn}} \mathrm{r}}{\mathrm{a}}\right)\right]$, where

$\operatorname{Sin}(m \theta)$ is used instead of $\operatorname{Cos}(m \theta)$ if $m>0$.

The values of $m$ and $n$ are integers and define the vibration modes, $J_{m}$ is the Bessel function of the first kind and $I_{m}$ is the modified or hyperbolic Bessel function of the first kind, a is the radius of the circular plate, and $\beta_{\mathrm{mn}}$ is the mode factor. The roots of equation (2) provide the allowed frequencies of vibration and are given as:

$$
\text { Frequency }=\frac{\pi \mathrm{h}}{2 \mathrm{a}^{2}} \sqrt{\frac{\mathrm{E}}{3 \rho\left(1-\sigma^{2}\right)}} \beta_{n \mathrm{~m}}{ }^{2} .
$$

The fundamental mode vibration with $\mathrm{m}=0$ and $\mathrm{n}=1, \mathrm{a}=0.032 \mathrm{~m}, \mathrm{~h}=0.00025 \mathrm{~m}, \rho=2.77 \mathrm{x} 10^{3} \mathrm{~kg} / \mathrm{m}^{3}$, Poisson's ratio $\sigma=0.33, \beta_{0}=1.015$, and modulus of elasticity $\mathrm{E}=73.1 \mathrm{GPa}$ is calculated to be approximately $1,223 \mathrm{~Hz}$ using equation (3) for the sample described in Figure 2. The predicted normalized fundamental mode displacement is shown in Figure 3.

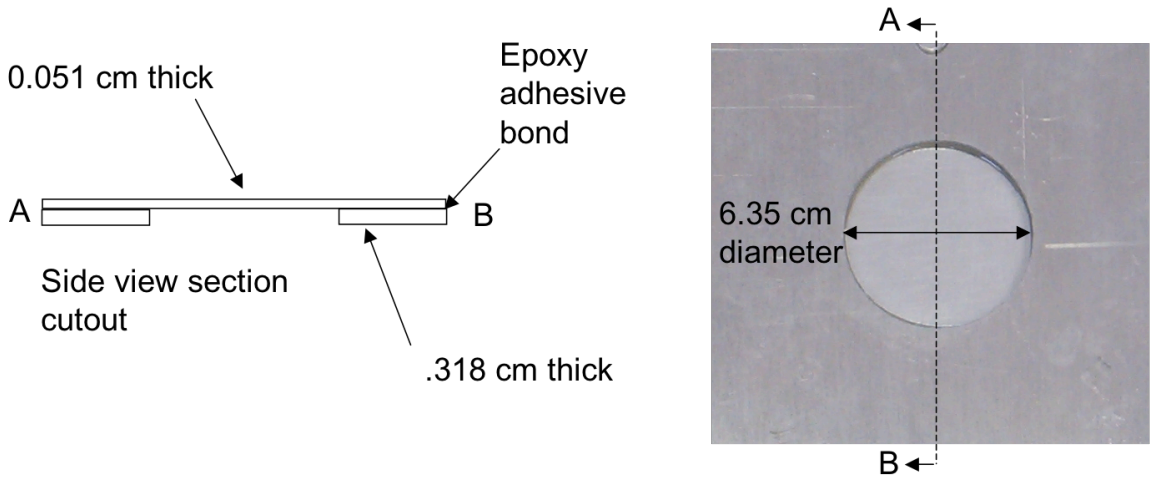

Figure 2. Drawing and picture of suspended circular plate sample. 


\section{COMPARISON OF MEASUREMENT RESULTS TO MODEL}

Using the system shown in Figure 1, the aluminum plate sample was tested with the camera frame rate set at $15 \mathrm{~Hz}$. Total number of images acquired was 2400 and the acoustic source was pulsed at $0.5 \mathrm{~Hz}$ for a total of 80 cycles. The suspended plate was driven into vibration using a sound pressure level (measured at 5 feet away) of approximately 110 dBA and the frequency was varied from $940-1,080 \mathrm{~Hz}$ in increments of $20 \mathrm{~Hz}$. The $0.5 \mathrm{~Hz}$ magnitude images as a function of frequency are shown in Figure 4. A resonance is detected at $1,020 \mathrm{~Hz}$ where significant heating is occurring. This temperature rise is due to the forced vibration from the acoustic source. The most likely mechanism of heating is internal friction. The real part (in-phase) image of the $1020 \mathrm{~Hz}$ magnitude image is shown in Figure 5 with a line plot over the defect area. It is interesting to note the in-phase image can be used to show relative displacement or movement. The brighter areas in this image reveal the areas of greatest displacement or in-phase heating. At the center and the edges is where there is minimal displacement and therefore less in-phase heating. The difference between the model predicted resonance of $1,223 \mathrm{~Hz}$ and the actual measured resonance of $1,020 \mathrm{~Hz}$ is due to the adhesive bond, which does not allow for perfectly rigid clamping at the boundary edges assumed in the model. These results verify the heating is due to the displacements caused by the acoustic source.

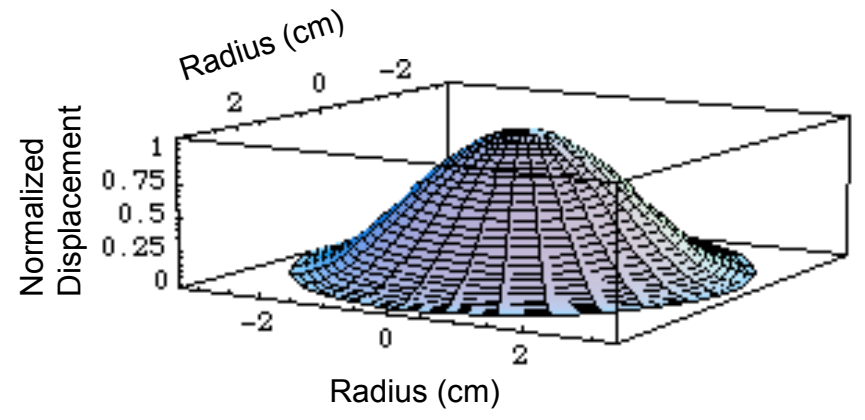

Figure 3. Fundamental mode vibration $\mathrm{m}=0, \mathrm{n}=1$.

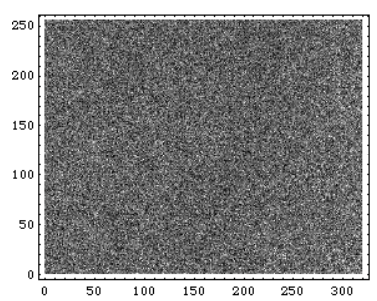

Excitation: $940 \mathrm{~Hz}$

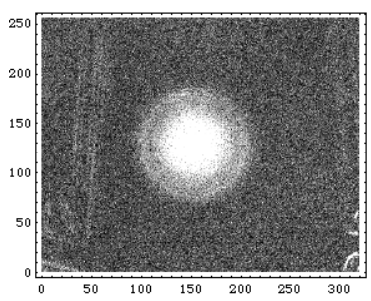

Excitation: $1020 \mathrm{~Hz}$

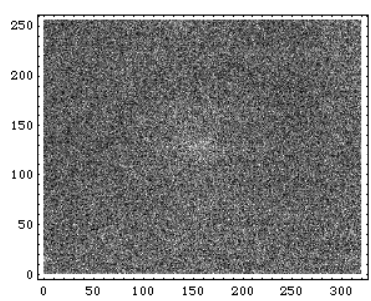

Excitation: $960 \mathrm{~Hz}$

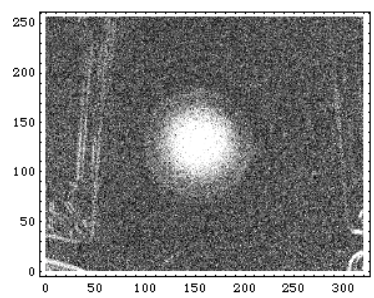

Excitation: $1040 \mathrm{~Hz}$

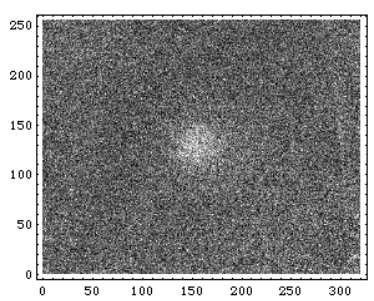

Excitation: $980 \mathrm{~Hz}$
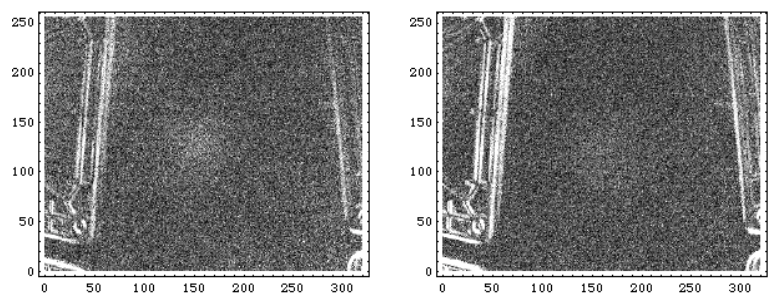

Excitation: $1060 \mathrm{~Hz}$ Excitation: $1080 \mathrm{~Hz}$

Figure 4. Resulting 0.5hz magnitude images using air coupled acoustic heating on aluminum plate. 

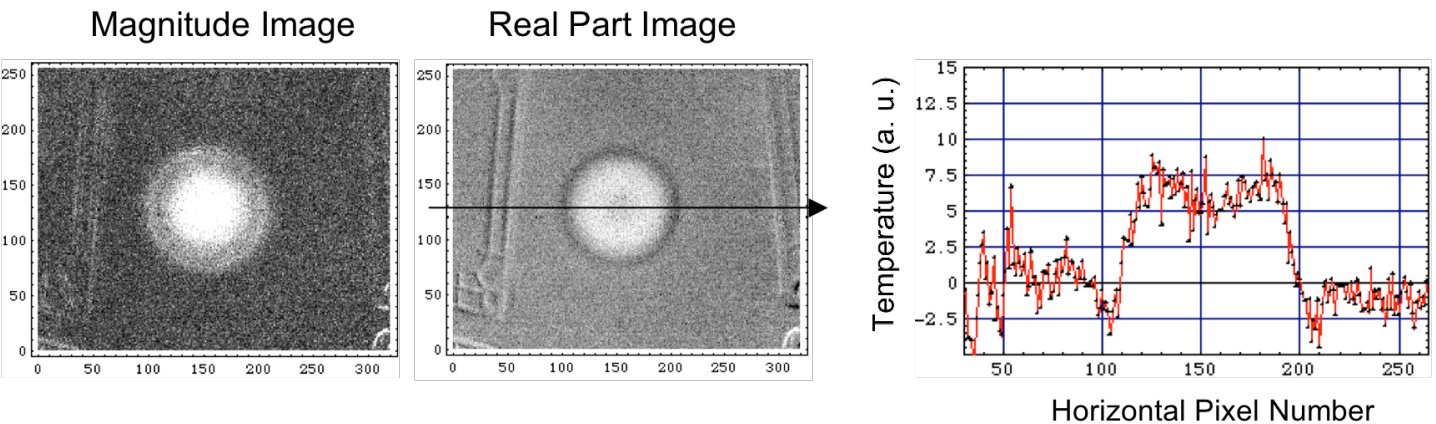

Figure 5. Real part of $0.5 \mathrm{~Hz}$ magnitude image at $1020 \mathrm{~Hz}$.

\section{MEASUREMENT RESULTS ON HONEYCOMB STRUCTURE}

The capability of the ACAT system was tested on a honeycomb sandwich structure with induced ballistic damage and the results are compared to conventional flash thermography. The sample, shown in Figure 6, is a full width AH-64 helicopter blade section with ballistic damage to the spar cells and its honeycomb trailing contour segment. The honeycomb segment is a sandwich structure consisting of 2 plies of co-cured glass epoxy fiberglass face sheets bonded to a lightweight Nomex ${ }^{\mathrm{TM}}$ core $(.64 \mathrm{~cm}$ cell size). The ballistic damage was induced by firing 0.22 caliber fragment simulated particles (FSP) at various velocities to achieve different levels of damage. Shown in Figure 6 is a conventional thermal inspection showing three areas with ballistic damage on the FSP exit side. Delaminations from the ballistic damage show up as light areas. Higher velocity projectiles fully penetrated the honeycomb segment producing a small hole and significant delamination and fiber breakage at the exit side. The sample was inspected with the ACAT system also shown in Figure 6. The acoustic source frequency was $1620 \mathrm{~Hz}$ pulsed at $0.5 \mathrm{~Hz}$. The corresponding acoustic thermography magnitude images at $0.00625 \mathrm{~Hz}$ and $0.5 \mathrm{~Hz}$ are shown in Figure 7. The 0.00625 $\mathrm{Hz}$ magnitude image reveals a much larger area of damage between the top two delaminated areas. This damage corresponds to a definite disbond, between the skin and core, resulting from a FSP impact from the lowest velocity that did not penetrate through yet provided enough energy to cause failure (disbond) between the skin and core. This area has reduced out of plane stiffness and therefore sound energy is able to vibrate this area resulting in a significantly higher temperature. Another interesting result is the $0.5 \mathrm{~Hz}$ magnitude image of Figure 7 where the image shows hot spots at the ballistic impact locations. This is again due to small delaminations in the outer skin weave cause by the impact event. The broken fiber/matrix allows the acoustic sound to more easily couple and vibrate thus acting as hot spots.

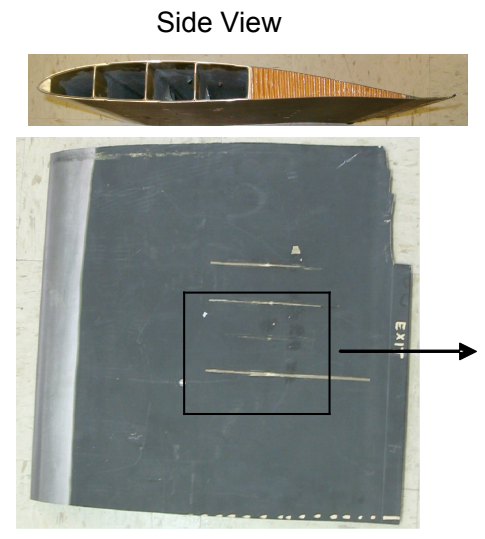

Top View of Blade Section

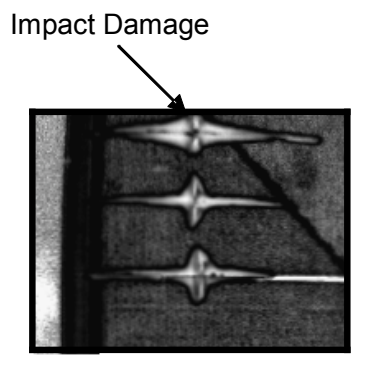

Conventional Thermal Inspection
Acoustic Thermography Image (magnitude image at $0.00625 \mathrm{~Hz}$ )

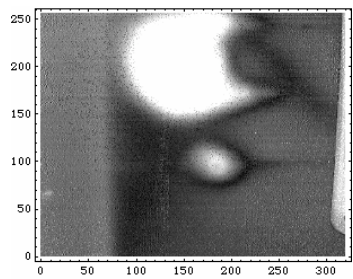

Figure 6. Helicopter blade structure with skin/core damage and corresponding flash thermography and ACAT inspection. 


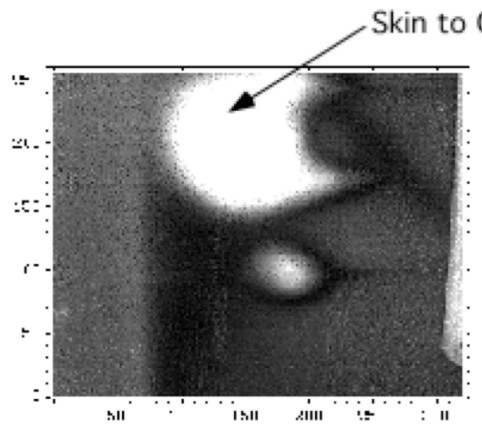

$0.00625 \mathrm{~Hz}$ Acoustic Image

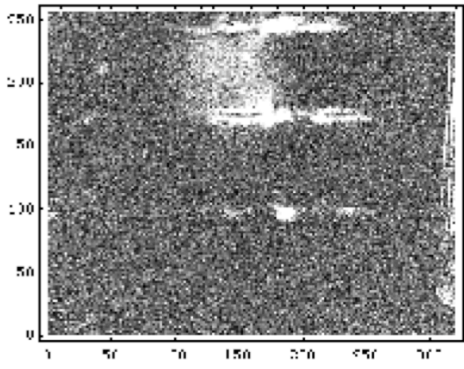

0.5 Hertz Acoustic Image

Figure 7. Acoustic thermography processed magnitude inspection images.

To verify the disbond detected between the skin and core, an edge cut on the blade sample was made along the core between the skin and core as shown in Figure 8. A comparison between conventional thermography and acoustic thermography images is also shown in Figure 8. The cut was made using a razor blade and was $4.4 \mathrm{~cm}$ long and penetrated approximately $1.9 \mathrm{~cm}$ into the core leaving some core material still attached to the skin. The cut area does not show up in the conventional thermography inspection image but clearly shows up (light area) using the acoustic technique. In addition, along the edge there appears to be additional damage. This small indication corresponds to some damaged core seen visually along the core edge.

To further evaluate the ACAT technique, honeycomb samples with a core defect were made. Defects were circular in shape and made in various diameters from $1.3 \mathrm{~cm}$ to $5.1 \mathrm{~cm}$ in size. To simulate damage from the blade it was found that fracture of the core can take place just below the meniscus level of the cured adhesive that bonds the core to the face sheets. This type of defect was simulated as shown in figure 9 where $.32 \mathrm{~cm}$ flat bottom holes were cored at the center of the $2.54 \mathrm{~cm}$ thick honeycomb sheet containing a $.32 \mathrm{~cm}$ cell size. In addition, core plugs were cut to the same size and placed in the corresponding hole lining up cell walls with that of the core as shown in figure 9. The face sheets (E-glass fabric) and film adhesive were added and the entire sample was vacuumed bagged and cured in an oven. The sample was then primed and painted aircraft black. Flash thermography

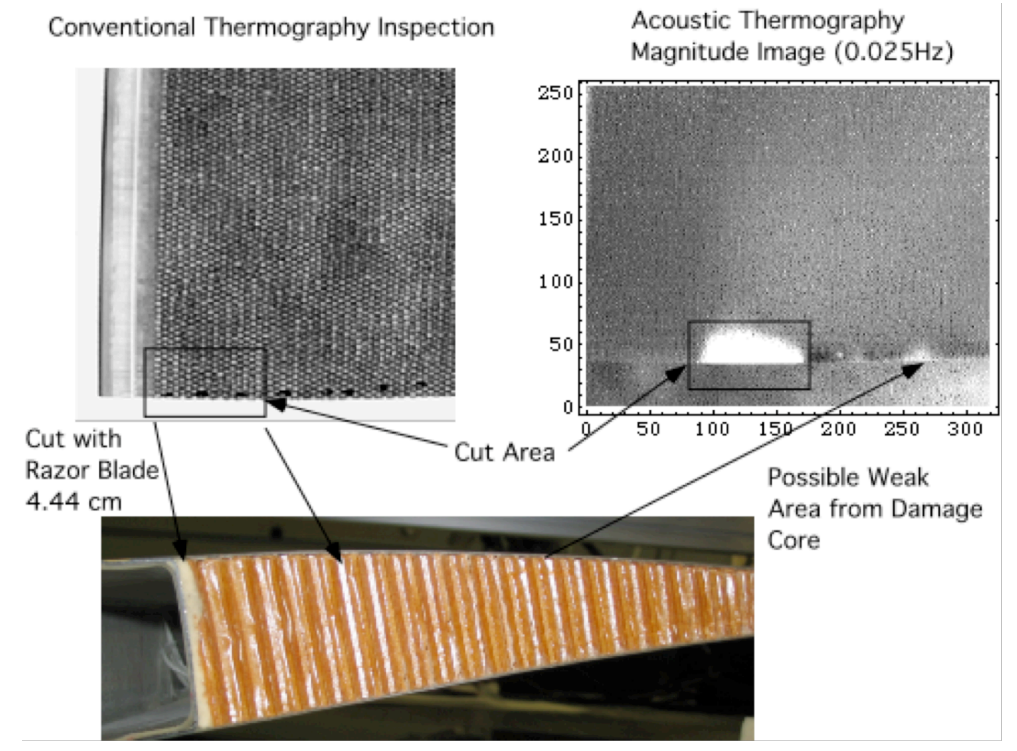

Figure 8. Side view of blade section with flash thermography and ACAT inspection results on cut core. 


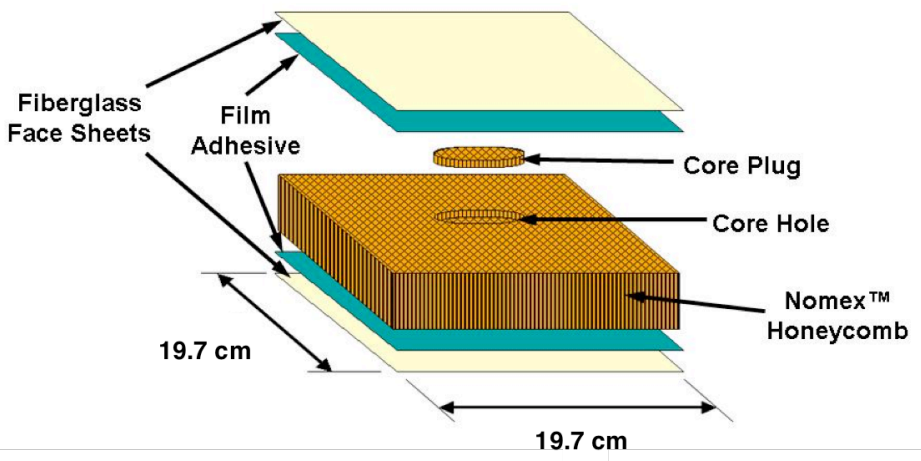

Figure 9. Illustration showing the assembly of the honeycomb core damage sample.

and preliminary ACAT results on the $5.1 \mathrm{~cm}$ diameter sample are shown in Figure 10. Taking into account the fiberglass face sheet with the attached core plug is free to vibrate, the fundamental mode vibration frequency with $\mathrm{m}=0$ and $\mathrm{n}=1, \mathrm{a}=0.0254 \mathrm{~m}, \mathrm{~h}=0.00032 \mathrm{~m}, \rho=1.94 \times 10^{3} \mathrm{~kg} / \mathrm{m}^{3}$, Poisson's ratio $\sigma=0.25, \beta_{01}=1.015$, and modulus of elasticity $\mathrm{E}=45 \mathrm{GPa}$ is estimated to be approximately $2,287 \mathrm{~Hz}$ using equation (3). The composite material properties are for unidirectional E-glass with a fiber volume fraction of $60 \%$ [5]. The ACAT inspection parameters were 2410 images acquired at $15 \mathrm{~Hz}$ with the acoustic source pulsed at $0.5 \mathrm{~Hz}$ and the frequency of the sound was $1700 \mathrm{~Hz}$. The resonant mode vibration is detected at a lower frequency as compared to the estimated model frequency. This is can be due to the rigid clamping requirements at the edges assumed by the model and also the diameter of the damage can appear larger due to the width of the core $(.32 \mathrm{~cm}$ cell size $)$ where the skin is still attached. These effects would decrease the resonant frequency. Using the ACAT system, skin to core disbonds, not detectable with conventional thermography, are detected as shown in Figure 10.
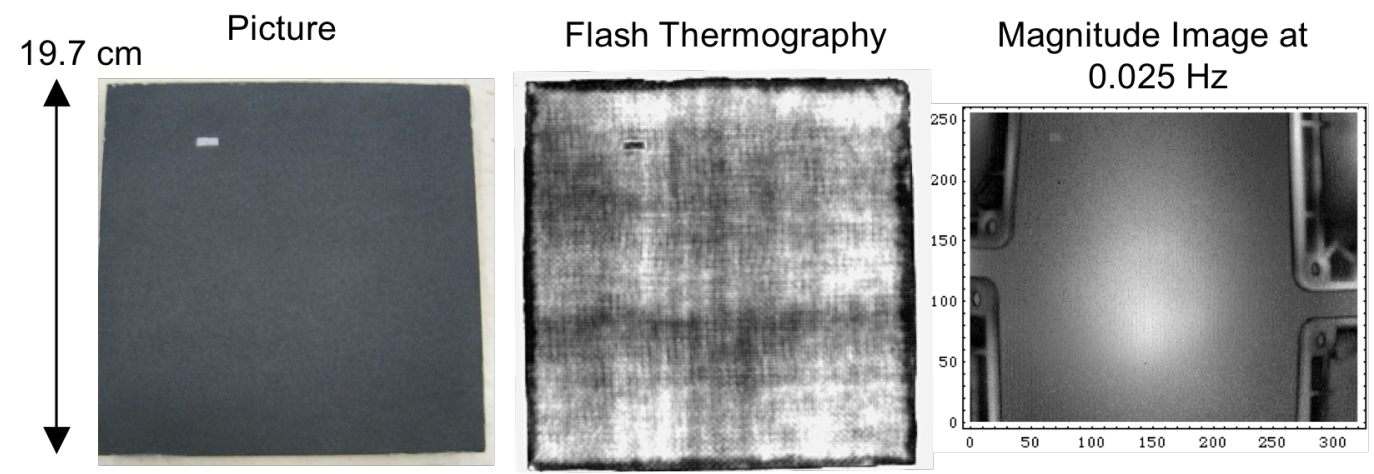

Figure 10. Conventional thermography and ACAT magnitude inspection image of cut core sample.

\section{CONCLUSIONS}

The air coupled acoustic source is a viable heating technique for thermal NDE of sandwich structures. A circular plate model was used to verify the acoustic coupling of the sound energy using the ACAT system. This acoustothermal technique has application for area inspection of core damage in composite honeycomb structures and can 
map out the damage areas. Selection of optimal heating frequency can perhaps be provided using a vibration sensor such as a laser vibrometer or accelerometer. In future work, the sensor signal and acoustic source could be used in a feedback loop. The vibration sensor can then be used to detect the resonant frequency. Finally for practical field application, further verification of the ACAT system is required with more testing of the fabricated honeycomb panels. Also a comparison of the ACAT technique to other inspection techniques is of interest.

\section{REFERENCES}

1. Robert F. Anastasi, Joseph N. Zalameda and Eric I. Madaras, "Damage Detection in Rotorcraft Composite Structures Using Thermography and Laser-Based Ultrasound”, SEM X International Congress \& Exposition on Experimental and Applied Mechanics, (2004).

2. A. Dillenz, G. Busse, and D. Wu, "Ultrasound Lockin Thermography: Feasibilities and Limitations", SPIE 1999.

3. M. Burke and W. O. Miller, "Status of VibroIR at Lawrence Livermore National Laboratory" in Thermosense $X X V I$, edited by D. D. Burleigh, K. E. Cramer, and G. Raymond Peakcock, Proc. Soc. of Photo-Opt. Instrumentation Eng. (SPIE), 5405, (2004) pp. 313-321.

4. P.M.Morse, Vibration and Sound, McGraw-Hill Book Company, Inc., (1948) pp. 208 -211.

5. ASM International, Composites Engineered Materials Handbook, Vol 1, (1987) page 178. 